\title{
Conocimientos adecuados de emergencias médicas: un problema de estudiantes y profesionales de la salud
}

\author{
Jane Chafloque-Carhuas, Mayra Pino-Delgado, Mayra Rivera-Paico, Cristian Díaz-Vélez
}

La Organización Mundial de la Salud [1] define la emergencia como aquella situación que tiene consecuencias inmediatas de riesgo para la vida, por lo que requiere atención inmediata. Según datos de la Cruz Roja Chilena [2], gracias a la intervención de personal capacitado en primeros auxilios, el $25 \%$ de personas afectadas se salvó de la muerte, el 38\% no quedó con secuelas como amputaciones, daños neurológicos o locomotores, principalmente, y sólo el $4 \%$ falleció. Cuando no se tiene un adecuado conocimiento al respecto, ello puede repercutir en la morbimortalidad de los pacientes que acuden a urgencias.

Mejía et al [3] realizaron un estudio para evaluar el nivel de conocimientos sobre emergencias médicas y encontraron que alrededor del $60 \%$ de los estudiantes obtuvieron puntuaciones que no alcanzaban el aprobado; aprobaron en mayor número los que recibieron algún curso sobre el tema y quienes realizaban prácticas en centros hospitalarios.

El tercer censo de infraestructura hospitalaria del año 2005 [4] reveló que Perú cuenta con 448 instituciones especializadas, hospitales y clínicas en el sector salud; de ello, los que se utilizan para desarrollar prácticas en pregrado y posgrado son sólo 247 (147 del MINSA, 79 de Essalud y 21 de las sanidades de las Fuerzas Armadas y Policiales). Además, según el Colegio Médico del Perú (www.cmp. org.pe), existen 166 médicos con especialidad en emergencias y desastres, por lo que en promedio no exista siquiera uno por hospital y en muchos casos las competencias son asumidas por médicos generales, internistas o de otras especialidades, sin una formación específica en ese ámbito.

Se sabe que el $24 \%$ de las atenciones en los hospitales peruanos corresponden a las realizadas en servicios de urgencias y emergencias [4]; en estudios realizados en profesionales de salud para medir los conocimientos sobre reanimación cardiopulmonar y cerebral llevados a cabo en Cuba no aprobó el 59\% [5], y otro estudio realizado en cinco ciudades cubanas encontró que no aprobaba el 75\% [6]. En una investigación más reciente que tenía como objetivo medir el conocimiento de los profesionales de salud sobre la nueva Guía 2010 de reanimación cardiopulmonar y atención cardiovascular de emergencia de la American Heart Association, se obtuvo como resultado que sólo el 1,4\% de médicos, el 1,4\% de obstetras y ninguna de las enfermeras conocía los cambios que presentaba la guía del año 2010 con referencia a su versión anterior [7].

Como se aprecia en el estudio de Mejía et al [3], una de las posibles explicaciones a este nivel inadecuado de conocimientos reside en el poco aprendizaje con el que se cuenta desde el pregrado. El 10 de agosto de 2009, el directorio del Consejo de Evaluación, Acreditación y Certificación de la Calidad de la Educación Superior Universitaria [8] aprobó el modelo de calidad para la acreditación de la carrera profesional universitaria de medicina, que consta de tres dimensiones, nueve factores, 16 criterios y 98 estándares. En la dimensión de formación profesional, dentro del factor de enseñanza-aprendizaje, se observa que el estándar 25 exige 'oportunidades educacionales disponibles en áreas multidisciplinarias, tales como medicina de emergencia, geriatría y disciplinas de ayuda al diagnóstico, como imaginología y patología clínica. Tal situación podría en cierta forma mejorar las perspectivas a largo plazo en los estudiantes de medicina, pero no olvidemos que en esta problemática también están involucrados los profesionales de la salud.

En estos profesionales, la explicación es similar a lo ocurrido en el pregrado. Cook et al [9] afirman que 'el conocimiento de los médicos comienza a deteriorarse en cuanto acaba su aprendizaje.' Hay que tener en cuenta que los libros de texto, los artículos de revistas y los colegas son las principales fuentes de información para los médicos, que quedan desfasadas enseguida, quizás por la falta de constante lec-
Sociedad Científica de Estudiantes de Medicina, SOCIEM-UNPRG (J. Chafloque-Carhuas, M. PinoDelgado, M. Rivera-Paico). Facultad de Medicina; Universidad Nacional Pedro Ruiz Gallo (C. Díaz-Vélez). Oficina de Inteligencia Sanitaria; Hospital Nacional Almanzor Aguinaga Asenjo (C. Díaz-Vélez). Chiclayo, Perú.

Correspondencia:

Dr. Cristian Díaz Vélez. Hospital Nacional Almanzor Aguinaga Asenjo. Plaza de la Seguridad, $\mathrm{s} / \mathrm{n}$. Chiclayo, Perú.

E-mail:

cristiandiazv@hotmail.com

(c) 2012 Educación Médica 
tura sobre información actualizada, argumentando falta de tiempo o de medios para hacerlo. Por tanto, la actualización de sus conocimientos cobra gran importancia.

\section{Bibliografía}

1. Organización Mundial de la Salud. El botiquín médico interinstitucional de emergencia. Geneva: OMS; 2006. URL: http://www.who.int/medicines/publications/web_iehk_sp.pdf.

2. Federación Internacional de Sociedades de la Cruz Roja y de la Media Luna Roja. URL: http://www.ifrc.org/sp/meetings/ events/firstaid03/world_news.asp. [12.09.2011].

3. Mejía CR, Quezada-Osoria C, Moras-Ventocilla C, QuintoPorras K, Ascencios-Oyarce C. Nivel de conocimientos sobre emergencias médicas en estudiantes de medicina de universidades peruanas. Revista Peruana de Medicina Experimental y Salud Pública 2011; 28: 202-9.

4. Ministerio de Salud del Perú (MINSA). Diagnóstico físico y funcional de infraestructura, equipamiento y mantenimiento de los hospitales e Institutos del Ministerio de Salud. Lima: MINSA; 2006. URL: http://www.minsa.gob.pe/dggdrh/ libros/pdf/s1/I-07.pdf
5. Machado-Álvarez MC, Roque-González CR, Barrios-Osuna CI, Nodal-Ortega J, Olive-González JB, Quintana-Pajón I. Nivel de conocimientos en reanimación cardiopulmonar cerebral en el Centro Nacional de Cirugía de mínimo acceso. URL: http://bvs.sld.cu/revistas/scar/vol 92 10/ane04210.htm.

6. López-Rodríguez MS, Navarrete-Zuazo V, Vallongo-Menéndez MB, Fernández-Abreu SM, De la Barrera-Fernández M, Ramírez de Arellano A. Estudio multicéntrico exploratorio sobre el nivel de conocimientos en reanimación cardiopulmonar y cerebral. URL: http://bvs.sld.cu/revistas/scar/vol5/no1/ scar05106.pdf.

7. American Heart Association. Aspectos destacados de las guías de la American Heart Association de 2010 para RCP y ACE. Arch Intern Med 2010; 17: 4-26.

8. Consejo de Evaluación, Acreditación y Certificación de la Calidad de la Educación Superior Universitaria (CONEAU). Modelo de calidad para la acreditación de la carrera profesional universitaria de medicina. Lima: Diario Oficial El Peruano; 25 de noviembre de 2009.

9. Cook DJ, Griffith LE, Sackett DL. Importance of and satisfaction with work and professional interpersonal issues: a survey of physicians practicing general internal medicine in Ontario. CMAJ 1995; 153: 755-64. 